\title{
PREPARATION OF SEEDS FOR MASS SCREENING
}

\author{
by \\ FLEMMING HELTVED', STEN AASTRUP ${ }^{2}$, OLUF JENSEN ${ }^{3}$, \\ GREGORY GIBBONS ${ }^{1}$ and LARS MUNCK'
}

\author{
1. Department of Biotechnology \\ 2. Department of Brewing Chemistry \\ 3. Department of Brewing Technology \\ Carlsberg Research Laboratory, Gamle Carlsberg Vej 10 \\ DK-2500, Copenhagen Valby
}

Keywords: Malt modification, preharvest, sprouting, Calcofluor, barley,

fluorescein dibutyrate.

An improved method for the rapid emplacement of 50 cereal seeds in a block of thermoplastic clay is described. The method is especially well suited for examination using fluorochromes after the seeds have been halved by abrasive sanding.

\section{INTRODUCTION}

Recently there has been a growing interest in the fields of malting and control of preharvest sprouting, to assess the internal physiological processes occurring in populations of individual seeds rather than obtaining data from bulked seed samples $(1,2,3,8,9)$. By analysing populations of seeds it has been possible to determine both the extent of internal changes related to germination and the variability within populations $(3,4,7)$.

The methods used have been based on four main parameters:

1) the extent to which barley endosperm can be washed out with warm water (10)

2) the permeability of the cereal endosperm to low molecular weight dyestuffs such as methylene blue $(5,9)$
3) the extent of barley endosperm cell wall breakdown as measured by the $\beta$-glucan specific stain Calcofluor $(3,6)$

4) most recently the detection in situ of enzymes produced during germination employing specific fluorochromes (8).

Preparation of seeds for these analyses has been carried out in a variety of ways.

The simplest method was to open the seeds by means of cutting with a razor blade or a scalpel and following treatment examining the half seeds laid out on a suitably coloured piece of background material $(9,10)$. Later a technique was devised in which the half seeds were imbedded by pressing into preformed holes in a block of plasticine (6).

At the same time a technique $(1,5)$ was described in which seeds were glued onto a glass 
plate using a rapidly hardening two component epoxy glue. Following hardening of the glue (from 15 min to 8 hours depending on the actual glue type used) the upper halves of the seeds could be removed by the abrasive action of a sanding machine prior to staining and examination.

This technique resulted in an analysis sample which had an excellent storage life and was easy to handle. The examination of the individual seeds was complicated however by the fact that, unless a great deal of time was taken in orienting each seed in the glue prior to hardening, the seeds lay randomly dispersed on the glass plate and were difficult to score.

In our laboratory, an attempt was made to overcome the orientation problem and at the same time avoid the epoxy glue, which we found slow and messy to work with. A vacuum system was employed for the orientation of 50 kernels prior to pressing them into a block of the thermoplastic clay "Cernit" (3). The clay was then hardened in an oven at $120^{\circ} \mathrm{C}$ for $20 \mathrm{~min}$ and sanded prior to staining and examination.

This paper describes the development of an improved method for secure, accurate and ordered emplacement of 50 cereal seeds into Cernit blocks.

In development of this method for routine analysis the following criteria were considered important:

a) The seeds should be arranged in rows with the longitudinal axes of the seeds in the same direction to allow for easy scoring of the individual seeds.

b) The preparation time involved should be as short as possible.

c) The seeds should be securely fastened in the block during the sanding procedure.

d) In cases where enzyme activity was to be measured the method should be usable without heat hardening of the Cernit block.

\section{MATERIALS AND METHODS}

\subsection{Plant material}

Samples of barley, wheat, rye and sorghum were commercially obtained.

Malted barley were either pilot malt made at the Carlsberg Research Center as previously de- scribed (2) or commercial malt obtained from the Carlsberg malthouse.

\subsection{Clay plate material}

Yellow non-toxic Cernit thermoplastic clay was purchased from $\mathrm{T}+\mathrm{F}$ Kunststoffe $\mathrm{GmbH}$, Rosenaustrasse 9, Dreieich, D-6072, West Germany, in $60 \mathrm{~mm} \times 80 \mathrm{~mm} \times 8 \mathrm{~mm}$ blocks custom extruded for Carlsberg.

\subsection{Analytical equipment}

The Carlsberg Seed Fixation System including press and accessories (Figures 1 - 12) was a commercial model obtained from the Carlsberg Research Center, Department of Biotechnology.

The abraded Cernit plates containing 50 half seeds were treated for cell wall identification according to AASTRUP et al. (3) and for lipase activity in barley, wheat, rye and sorghum according to JENSEN and HeLTVED (8).

The preparations were evaluated for cell wall breakdown in the Malt Modfication Analyser System Carlsberg and for diffussion of hydrolytic enzymes in the Seed Quality Analyser-System Carlsberg which were commercially obtained from the same supplier as the press unit.

\subsection{Sample preparation by use of the Carlsberg seed fixation system}

The seed alignment system consists of a plastic overmatrix (Figure 1) and an aluminium alloy undermatrix (Figure 2) both supplied with 50 seed holes.

The overmatrix is placed upon the undermatrix and the seed sample is tipped into the overmatrix (Figure 3). Following gentle shaking on an inclined plane the excess seeds fall off and 50 seeds remain in the overmatrix holes (Figure 4). The overmatrix is carefully removed with the 50 seeds in place on the undermatrix (Figure 5).

The seeds are now pressed into the custom extruded block of Cernit using a specially constructed press as follows. The undermatrix with seeds is placed under the press plate (Figure 6).

The block of Cernit is pressed into the Cernit holder (Figure 7), the excess plastic foil is removed (Figure 8) and the Cernit holder slid into place in the press (Figure 9). The Cernit holder is fixed magnetically and thus remains in place during the next step in which the seeds are pressed up 

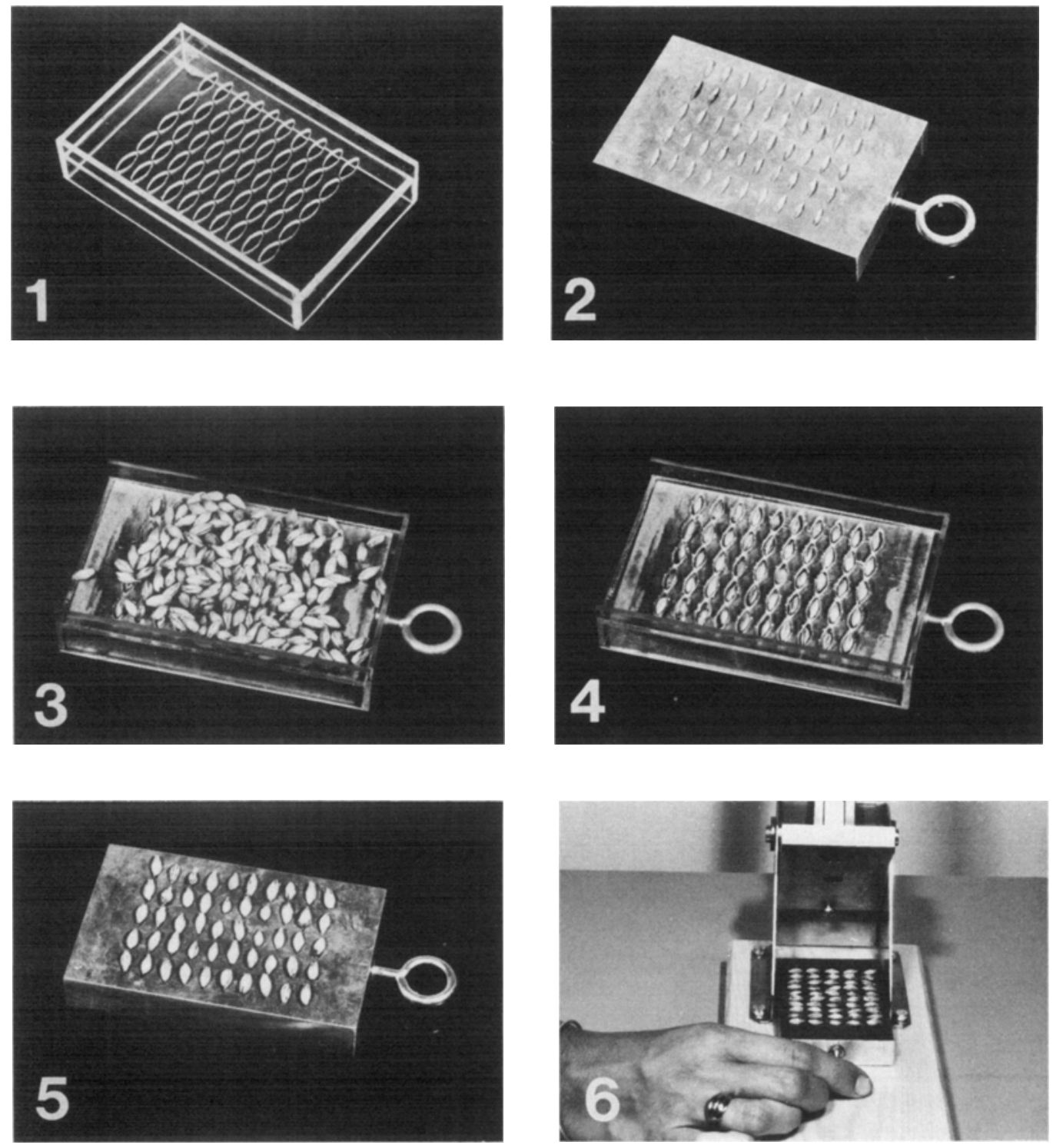

Figure 1. Plastic overmatrix with holes for 50 barley malt kernels.

Figure 2. Aluminium undermatrix with holes for 50 barley malt kernels.

Figure 3. Placement of sample on the overmatrix mounted on the undermatrix.

Figure 4. Appearance after shaking off the excess seeds.

Figure 5. Appearance of undermatrix after removal of overmatrix.

Figure 6. The undermatrix with seeds placed in the press unit. 

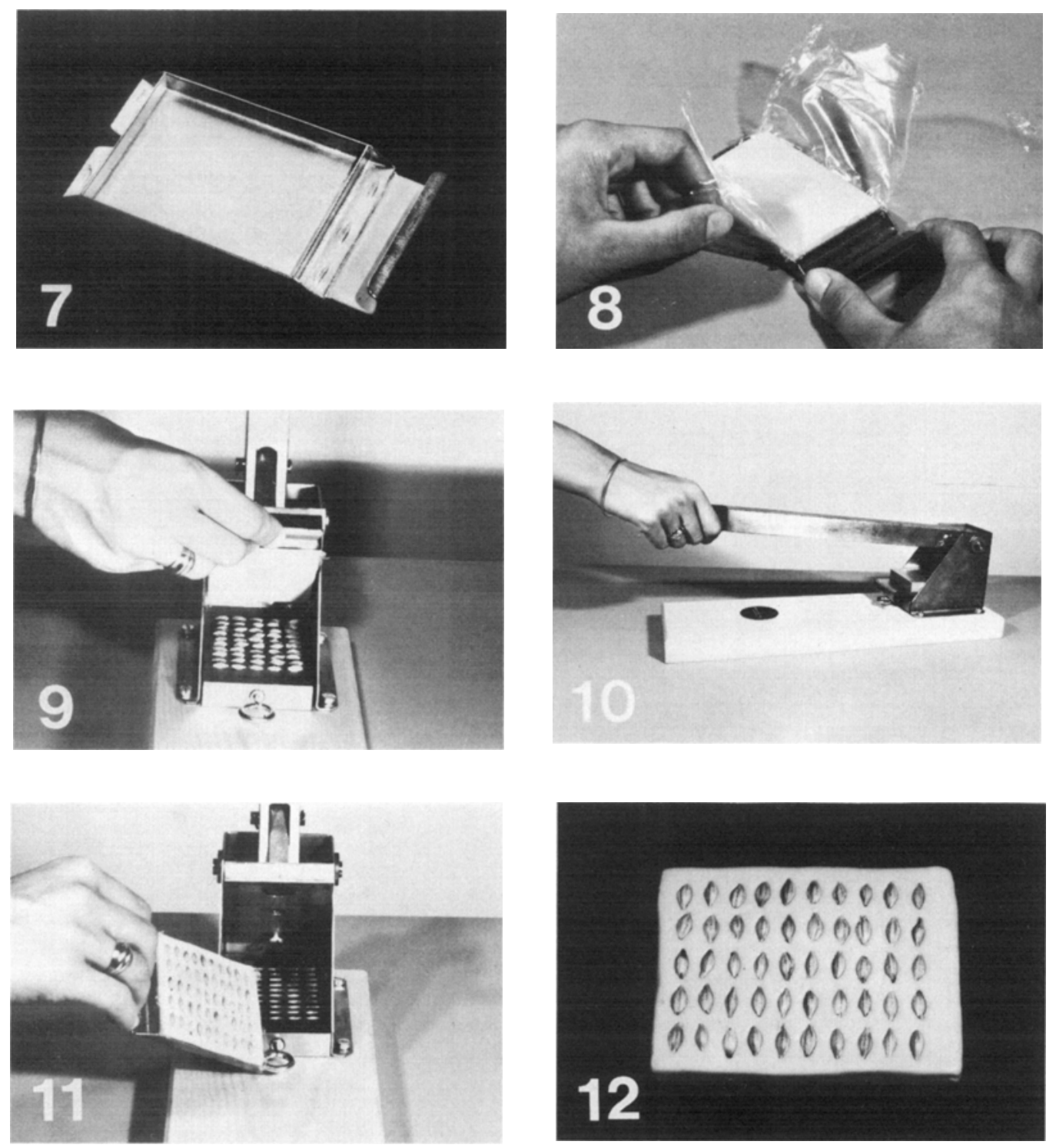

Figure 7. The Cernit holder. The sides of the holder have a $6^{\circ}$ angle to facilitate removal of the Cernit block.

Figure 8. Removal of the plastic foil from the exposed surface of the Cernit block.

Figure 9. Placement of the Cernit into the magnetic holder of the press.

Figure 10. Pressing of the seeds into the Cernit for $1-2$ seconds.

Figure 11. Removal of the Cernit block and holder.

Figure 12. Appearance of the unhardened block containing 50 ordered seeds.

into the Cernit (Figure 10). The press time has been found to be optimal between 1 and 2 seconds.

After pressing, the Cernit holder can be removed (Figure ll) and the block containing the 50 seeds (Figure 12) treated as required.

\subsection{Hardening}

\subsubsection{Heat hardening}

The thermoplastic Cernit clay can be hardened at any temperature between $90^{\circ} \mathrm{C}$ and $150^{\circ} \mathrm{C}$ for $10 \mathrm{~min}$. Temperatures above $150^{\circ} \mathrm{C}$ are not recommended by the manufacturer. 
A microwave oven can also be used to harden the blocks. A setting of $400 \mathrm{~W}$ for $2 \mathrm{~min}$ followed by $1-2$ min cooling and a further minute at $400 \mathrm{~W}$ has been found to be optimal. Higher wattage results in a severe bubbling of the Cernit clay.

Following hardening, the blocks are allowed to cool for $8 \mathrm{~min}$ at $25^{\circ} \mathrm{C}$ or $2 \mathrm{~min}$. at $-18^{\circ} \mathrm{C}$ prior to sanding.

\subsubsection{Cool hardening}

In cases where measurement of heat labile enzyme activity is required the heating technique described above cannot be used.

The block can be hardened in such cases by freezing prior to sanding. A 20 hours freezing at $18^{\circ} \mathrm{C}$ or when extremely rapid hardening is required, 8-10 sec in liquid nitrogen, is sufficient to harden the blocks.

\subsection{Sanding}

Kernels are best sanded using a sanding machine mounted in a vice (Figure 13). The clay plate with kernels is pressed gently down against the sandpaper for $30-45 \mathrm{sec}$. We have found that for field samples of cereal seeds grade 60 sandpaper is ideal. For malt samples grade 80 is suitable (Figure 14).

It is also possible to sand the seeds without hardening the Cernit clay, if care is taken during the sanding operation.

\subsection{Storage of sanded samples}

Samples, heat hardened prior to examination, can be preserved for at least 2 years by a simple coating of the Calcofluor stained seeds with a non

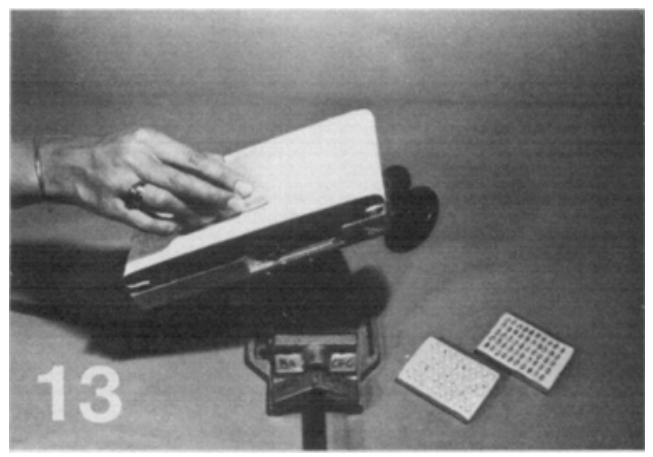

-fluorescent varnish or clear nail polish/acetone (2:1) mixture.

Samples examined by the fluorescein dibutyrate method (8) can be heated to $120^{\circ} \mathrm{C}$ for 20 min following staining, varnished as described above and stored in the dark at $25^{\circ} \mathrm{C}$. The fluorescein is stable in the dark for at least 4 months.

Unhardened samples can also be stored at -18 ${ }^{\circ} \mathrm{C}$ for at least 4 months.

\section{DISCUSSION}

The present Carlsberg seed fixation system produces conveniently sets of 50 longitudinally abraded half seeds on a Cernit plate. Without hardening it takes about $5 \mathrm{~min}$ to prepare the seeds for analysis. The specially designed press ensures a secure embedding and accurate positioning of the seeds in the Cernit clay. The ordering of the seeds in rows facilitates description of the sample.

The application of this method to barley malt has been described in this paper. Successful experiments on the fixation and sanding of wheat, rye and sorghum have been performed in this laboratory using over- and undermatrixes designed for each application.

Thus, this present seed fixation system fulfills the aims mentioned previously and represents a drastic improvement in both the time and the ease of performing single seed analysis of cereals.

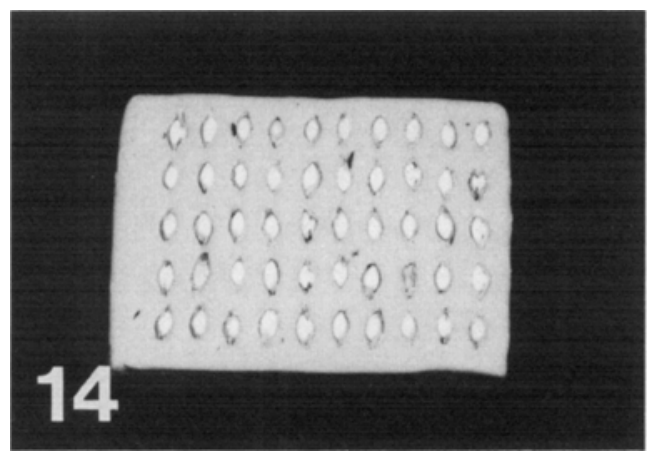

Figure 13. Sanding of the exposed upper halves of the seeds with an orbital sander.

Figure 14. Appearance of a sanded malt sample. 


\section{REFERENCES}

1. Aalbers, V.J.: Improved method for the determination of vitreosity in malt. Brewers Digest LV, 8, 38-39 (1980)

2. Aastrup, S. \& K. ERdal: Quantitative determination of endosperm modification and its relationship to the content of 1,3:1,4- $\beta$-glucans during malting of barley. Carlsberg Res. Commun. 45, 369-379 (1980)

3. Aastrup, S., G.C. Gibbons \& L. Munck: A rapid method for estimating the degree of modification in barley malt by measurement of cell wall breakdown. Carlsberg Res. Commun. 46, 77-86 (1981)

4. Carnielo, M., M.-A. Foucaut \& M. Moll: Application of a test for modification in green and kilned malts. Brauwissenschaft 35, 168-170 (1982)

5. Drost, B.W., V.J. Aalbers \& L. Pesman: Prediction of brewing quality by malt modification determination. European Brewery Convention, Monograph VI, 224-234 (1981)
6. GibBons, G.C.: Visualization of $\alpha$-amylase movement and cell wall breakdown during barley malting - Practical application of current research. J. Amer. Soc. Brewing Chemists. 39, 55-59 (1981)

7. GibBons, G.C. \& E.B. Nielsen: New analyses in malting and brewing. J. Inst. Brew. In press (1982)

8. Jensen, S.AA. \& F. Heltved: Visualization of enzyme activity in germinating cereal seeds using a lipase sensitive fluorochrome. Carlsberg Res. Commun. 47, 297-303 (1982)

9. KRINGSTAD, H.: Use of colour indicators as tests for the modification of malt. Proc. European Brewery Conv. Congress, Interlaken, 131138 (1969)

10. Palmer, G.H.: A method for direct assessment of malt modification. J. Inst. Brew. 81, 408-409 (1975) 